WIENER SLAVISTISCHES JAHRBUCH, Band 53/2007, 229-239

(C) 2007 by Österreichische Akademie der Wissenschaften, Wien

K A T J A S T U R M - S C H N A B L

\title{
Miklosichs Bedeutung für die Slowenistik unter besonderer Berücksichtigung seiner Lesebücher für Mittelschulen
}

\begin{abstract}
Franc Miklošič je kot ustanovitelj in prvi profesor katedre za slovanske jezike na dunajski univerzi tudi svojo slovensko materinščino, njeno literaturo in kulturo, vključil v svoje znanstveno delo. Od vsega začetka se je zavzemal za tri narodno in kulturnopolitično pomembne projekte: za slovenske čitanke za gimnazije, za strokovni slovensko-nemški slovar in slovar narodnega jezika ter za slovensko slovnico. Miklošič sam je prevzel redakcijo čitank za višje razrede gimnazije, ki jasno pokažejo urednikovo težnjo po kodificiranju slovenskega knjižnega jezika, odlikuje pa jih tudi pedagoško premišljena in pestra vsebinska ponudba. Njegovo gradivo za slovensko-nemški slovar se je na koncu zlilo v Pleteršnikov slovar, po njegovi slovenski slovnici, ki jo je izdelal za Primerjalno slovnico slovanskih jezikov, pa so A. Janežič, J. Šuman in drugi sestavili slovnice za šolsko rabo.
\end{abstract}

Franz Miklosichs Beitrag zur Entwicklung der Slowenistik ist gleichzeitig eine der großen gesellschafts- und kulturpolitischen Leistungen im habsburgischen Raum in der zweiten Hälfte des 19. Jahrhunderts. Miklosich schuf die Grundlagen für die Slowenistik, als er die Slawistik als universitäres Forschungsfach schuf, indem er als Politiker, d. h. Reichstagsabgeordneter den zuständigen Minister Leo Thun ${ }^{1}$ davon überzeugte, dass die Einrichtung einer Lehrkanzel für slawische Philologie und Literatur eine gesellschafts- und kulturpolitische Notwendigkeit war. Unter Slawistik verstand er die wissenschaftliche Behandlung und Affirmation jeder einzelnen slawischen Sprache, Literatur und Kultur in ihrer individuellen Selbstständigkeit. Dies war damals in der Philologie ein ganz neues, demokratisches Denkmodell. In einer Festrede vor deutschen Philologen und Schulmännern, anlässlich der Eröffnung ihrer jährlichen Versammlung, die im Jahre 1858 in Wien stattfand, bestätigt und betont er seine Ansicht mit den folgenden Worten:

${ }^{1}$ Graf Leo Thun-Hohenstein (1811-1863) war von 1849 bis 1860 Minister für Cultus und Unterricht. Miklosich war einer seiner engsten Mitarbeiter bei der großen Bildungsreform in dessen Amtszeit. Es mag kein Zufall sein, dass Thuns Standbild im Arkadengang der Universität Wien umgeben ist von den Denkmälern jener Männer, die seine Reformen erarbeitet haben: Franz Miklosich, Hermann Bonitz, Franz Exner und Leopold Hasner. 
Wenn die Philologie im allgemeinen das Leben eines Volkes in einem abgeschlossenen Zeitraume nach allen seinen Richtungen wissenschaftlich zu erforschen strebt, so gibt es so viele Philologien, als es verschiedene Völker gibt, deren literarische Denkmäler zur Erkenntnis des Lebens in seiner idealen und realen Richtung ausreichen. Man kann daher, wenn man sich auf Europa beschränkt, der classischen Philologie die germanische, romanische und slavische entgegensetzen. ${ }^{2}$

Das heißt, dass natürlich auch seine eigene Muttersprache, deren Literatur und Kultur zum Gegenstand seiner wissenschaftlichen Forschung wurden; viel mehr noch, als politisch engagierter Mensch war er bestrebt, die Ergebnisse seiner wissenschaftlichen Forschungen gesellschaftspolitisch umzusetzen und für das slowenische Bildungssystem und die slowenische Intelligenzia nutzbar zu machen. So sind auch Miklosichs intensive und beständige Bemühungen um slowenische Lesebücher, Grammatiken und Wörterbücher zu verstehen. Konkret sollen hier drei Projekte angesprochen werden, die Miklosichs Anstrengungen und Unternehmungen für die Entwicklung seiner slowenischen Muttersprache zur Sprache der künftigen slowenischen Intelligenzia und als Forschungsgegenstand an der Universität klar aufzeigen. Dies sind die slowenischen Lesebücher für die Gymnasien, ein umfassendes, auf wissenschaftlicher Basis aufbauendes slowenisch-deutsches Wörterbuch und schließlich eine Grammatik der slowenischen Sprache, die in ihrer Ausführung in der Vergleichenden Grammatik der slavischen Sprachen blieb, dann aber zum Modell für die slowenischen Schulgrammatiken eines Anton Janežič (1828-1869) oder Josip Šuman (1838-1908) wurde.

Bereits im Jänner 1849 berichtet Miklosich seinem Freund, dem Volksaufklärer Jožef Muršec (1807-1895) in Graz, dass er für das Ministerium Vorschläge für das slowenische Schulwesen ausarbeite (Sturm-Schnabl 1991: 67-68). ${ }^{3}$ Im April war er

Aus Miklosichs Festrede bei der Eröffnung der 18. Versammlung deutscher Philologen,
Schulmänner und Orientalisten in Wien, in: Verhandlungen der achtzehnten Versamm-
lung deutscher Philologen, Schulmänner und Orientalisten in Wien vom 25. bis 28. Sep-
tember 1858 . Wien 1859 . Welche kongeniale Vision Miklosich und Thun von einer de-
mokratischen, gleichberechtigten Koexistenz der Völker und Kulturen hatten und welchen
Stellenwert sie der Produktion von Mehrwert durch Interferenzen und gegenseitige Be-
fruchtung beimaßen, mag ein Auszug aus Thuns Eröffnungsrede bei der nämlichen Ver-
anstaltung veranschaulichen (S. XI): „Es gibt keinen Staat in Europa, in welchem so viele
bildungsfähige Völker wohnten, als Oesterreich, wo die Gesetze in zehn Sprachen kund-
gemacht, Schulbücher, und zwar nicht nur für Volks-, sondern theilweise selbst für Mit-
telschulen, in zehn Sprachen verfasst und gedruckt werden. Jeder Volksstamm hängt mit
Begeisterung an seiner Sprache, und ein nicht geringer Theil der geistigen Bewegungs-
kraft Oesterreichs liegt in dieser naturgemäßen Begeisterung. Soll sie höheren Zwecken
dienlich sein, so muss ihr geistige Nahrung geboten werden, und dies muss zunächst
durch gründliche philologische Studien geschehen. [...] Die tiefere Einsicht in die unver-
wüstliche Naturkraft, die jeder lebenden Sprache innewohnt, und die Erkenntnis des stei-
genden inneren Werthes der Erzeugnisse der heimischen Literatur wird den Gemüthern
jene Beruhigung gewähren, die erforderlich ist, damit verschiedene Sprachen friedlich ne-
ben einander bestehen.“
In diesem Brief vom 9 . 1 . 1849 aus Kremsier an Jožef Muršec charakterisiert Miklosich
die steirischen Abgeordneten Dominkus, Krajnc, Supanz, Smreker und Sturm im Reichs-
tag von Kremsier vor allem hinsichtlich ihrer politischen und nationalen Zuverlässigkeit 
Professor für slavische Philologie und Literatur geworden (seine slavistischen Forschungen und Publikationen sowie seine Lehrtätigkeit laufen voll an). Im November 1849 bekam Miklosich den offiziellen Auftrag des Ministeriums slowenische Lesebücher für Gymnasien zu verfassen (Sturm-Schnabl 1991: 69-70). ${ }^{4}$ Er selbst übernimmt die Lesebücher für die Oberstufe, jene für die Unterstufe übernimmt Janez Bleiweis in Ljubljana. Dies waren die ersten slowenischen Schulbücher für Mittelschulen.

Während der Arbeit an den Lesebüchern stehen beide Verfasser ${ }^{5}$ in regem Briefkontakt bzw. diskutieren die Frage der Sprache. Miklosich hat ein klares Konzept, nämlich die Schriftsprache möglichst an die Volkssprache anzulehnen, ${ }^{6}$ was durchaus im Sinne Jernej Kopitars war, der dieses Prinzip bereits an Vuk Stefanović Karadžić, den Reformator der serbischen Schriftsprache, vermittelt hatte. In der Frage der Schrift aber hat Miklosich einen großen selbstständigen Schritt nach vorne getan. Seit der Zeit des ABC-Streites bei den Slowenen war die Schreibweise noch immer nicht konsolidiert (Francè Prešeren hatte für das Manuskript seiner Sammlung Poezije 1847 noch nicht die Gajica angewendet). Miklosich aber verwendet in den Lesebüchern konsequent und ohne weitere Diskussionen die Gajica, die sich dann bei den Slowenen durchsetzt. Seine vier Lesebuchbände erscheinen 1853, 1854, 1858 und $1861\left({ }^{2} 1881\right)$, und schon im ersten Band bietet der Miklosich neben slowenischen Originaltexten und Autoren auch Übersetzungen aus dem Französischen, Polnischen, Tschechischen, Deutschen, Russischen, Kroatischen, Serbischen, Altgriechischen, Lateinischen. Hier publiziert Miklosich auch seine eigene Reisebeschrei-

und stellt fest, dass die drei letztgenannten für die slowenische Sache kaum nützlich seien. Miklosich fragt Muršec nach seinen Ansichten über das slowenische Schulwesen und teilt mit, dass er für das Ministerium in dieser Frage Vorschläge ausarbeite, denn das gegenwärtige Ministerium sei der slowenischen Sache geneigt. Er bittet Muršec für dieses Unternehmen die Unterstützung durch die gesamte slowenische Gesellschaft zu organisieren.

4 Miklosich an Jožef Muršec 14. 11. 1849 aus Wien. Miklosich bedankt sich für einen Brief und das Vertrauen. Er sei gerne bereit mit Minister Thun in der bekannten Angelegenheit persönlich zu sprechen, sofern dies aus zeitlichen Gründen durchführbar sein werde. Die Arbeit am Reichsgesetzblatt habe er zurückgelegt und an Cigale übertragen. Das altslovenische Wörterbuch habe er beendet; er ersucht Muršec, ihm beim Verkauf des Buches behilflich zu sein und u. a. auch Kvas zu informieren. Er beabsichtige ein slowenisches Lesebuch für Gymnasien zu verfassen, wofür er ebenfalls um die Unterstützung Muršecs und anderer Slowenen bitte; solange es keine entsprechenden Lehrbücher gebe, sei das Ministerium nicht bereit Slowenischlehrer anzustellen.

5 Die Bleiweis'sche Reihe erschien 1850-1855 und hieß Berilo slovensko und war für die Unterstufe der Gymnasien bestimmt.

6 Von Juli 1853 bis Oktober 1854 sind 4 Briefe zwischen Miklosich und J. Bleiweis erhalten, in denen die beiden über auftauchende Probleme bei der Erstellung der Lesebücher behandeln. Es sind dies die Briefe Nr. 29, 30, 35 und 36 (Sturm-Schnabl 1991: 92-94, 100-102). So schreibt Miklosich am 22. Juli 1853, dass er sich bemüht habe, möglichst wenig von der slowenischen Volkssprache abzuweichen und dass er überzeugt sei, Bleiweis habe das ebenfalls so gehalten. Im Schreiben vom 10. Oktober 1854 meint Bleiweis, Miklosich sollte in der Frage der Verbalaspekte eingreifen. Es werden auch die Inhalte der beiden Reihen koordiniert und sprachliche Fragen abgestimmt. 
bung über Konstantinopel (Slovensko Berilo 1853: 146-150). Deutlich liegt dem Konzept der Wille zugrunde, den Schülern über den Lesestoff auch Allgemeinbildung zu vermitteln. Mit diesen Lesebüchern für die Oberstufe der Gymnasien hat Miklosich einen Maßstab für das Niveau slowenischer Schullesebücher gesetzt, das später z. B. auch ein Jakob Sket bei seinen Ausgaben eingehalten hat und das den Schülern neben der Maitrise der slowenischen Sprache auch ein hohes multikulturelles Niveau an Allgemeinbildung vermittelte, zugleich aber eine historisch-slowenistische Grundausstattung bot.

Die Titelseite des Lesebuches für die fünfte Gymnasialklasse lautet: Slovensko berilo za peti gimnazijalni razred. Izdal Dr. Fr. Miklošič. Velja 24 kr. C. M. na Dunaju. V zalogi ces. kralj. bukev za šole pri Sv. Ani v Janezovih ulicah 1853. In diesem ersten Band seiner Reihe hat Miklosich u. a. Matevž Ravnikar, Anton Martin Slomšek, Matija Valjavec, Ivan Navratil, Matija Cigale, Franc Metelko, Janez Trdina, Janez Bleiweis als Mitarbeiter, sei es für Übersetzungen oder Originaltexte, herangezogen. Einige Beiträge seien hier genannt, um einen konkreteren Eindruck von der Struktur des Lesebuches zu vermitteln. So zählt auch der berühmte slowenische Missionar Friderik Irenej Baraga (1797-1868) zu den Autoren dieses ersten Bandes. Baraga wirkte unter den Indianern in Nordamerika, wurde 1853 Bischof in Cincinnati und später in Marquetu/Michigan. Er schrieb religiöse Schriften und Übersetzungen auch in Indianersprachen, u. a. verfasste er eine Grammatik und ein Wörterbuch der Sprache der Chippewas. In deutscher Sprache erschien 1837 das Buch Geschichte, Charakter, Sitten und Gebräuche der nord-amerikanischen Indier. Theils aus zuverlässigen Quellen, theils aus eigener Erfahrung gesammelt und herausgegeben von Friedrich Baraga, Missionär der Dtschipwe=Indier am See superior, im Inneren von Nord=Amerika. Laibach 1837. Gedruckt bei Joseph Blasnik. Verlegt und zu finden bei Johann Klemens, bürgerl. Buchbinder. ${ }^{7}$ Miklosich nahm in den

${ }^{7}$ Dieses Buch erschien in französischer Übersetzung unter dem Titel: Abrégé de l'Histoire des Indiens de l'Amerique septentrionale, par F. Baraga, Missionaire au lac Supérieur. Traduit de l'Allemand [rule] Paris, A la Société des Bon Livres rue des saints-peres, 1837 Paris. - Imprimerie de E. Bailly, Place Sorbonne, 2. Ebenfalls im Jahr 1837 erschien es in slowenischer Übersetzung mit dem Titel: Popis navád in sàdershanja Indijanov Polnozhne Amerike. Spisal v nemshkim jesiku Friderik Baraga, misijonar per Ozhipve - Indijanih na otóku Gorenjiga Jezera, v’ sredi Polnozhne Amerike. V' Ljubljani, natisnil Joshef Blasnik, 1837. Na prodaj pri Janesu Klemensu. Eine Neuauflage veranstaltete 1970 die Mohorjeva družba in Celje unter dem Titel: Zgodovina, značaj, nravi in šege severnoameriških Indijancev. Von Baragas slowenischen erbaulichen Büchern erschien seine Gebetssammlung: Dušna paša za kristjane, ki žele v duhu in resnici moliti Boga. Spisal Friderik Baraga, Kaplan v Metliki. V' natis tih bukev so milostlivi Firsht Gospod Anton Alois, Ljubljanski Shkof 20. grudna 1828 dovolili. Bis 1905 erschienen mindestens 18 Ausgaben. Die Ausgabe von 1905 erschien bei Hermagoras/Mohorjeva družba in Klagenfurt/Celovec und Baraga wird als „bivši škof v Severni Ameriki“ apostrophiert. Dieses Werk gilt als das beste und adäquateste Gebetsbuch bei den und für die Slowenen. Baragas Wirken unter den Indianern hatte sehr starke soziale Aspekte; neben der Tatsache, dass er sich um ihre Sprache bemühte, kämpfte er auch gegen Alkoholismus und Diskriminierung (vgl. Ceglar 1991). 
ersten Band des Lesebuches Baragas authentischen Bericht Nekoliko iz kratkega popisa zgodb Indijanov Polnočne Amerike über die frühen europäischen Siedlungen, die ersten friedlichen Begegnungen und kriegerischen Auseinandersetzungen mit den Indianern auf, im dem auch die Geschichte der Indianerin Pokahontas und John Smith' erzählt wird (Slovensko Berilo 1853: 27-35). Einen angemessenen Platz erhält Francè Prešeren mit Slovo od mladosti, Zabavljica, Matiju Čopu und Nuna. J. Navratil lieferte eine Kurzbiographie zu Matija Čop wie auch zu Dositej Obradović, der zudem mit einer Fabel vertreten ist. J. Koseski ist $u$. a. mit der Übersetzung von Schillers Kranichen des Ibikus präsent. M. Cigale übersetzte einen juridischen Text nach Montesquieu. Offenbar war dem Herausgeber daran gelegen, Kärnten und die Steiermark historisch zu dokumentieren, ebenso die Konfrontation der Slowenen mit den Türken, die zudem mit der Volksballade Ženitba Matjaža kralja belegt wird. Natürlich wird auch Maria Theresia gewürdigt, wie überhaupt historische und humanistische Aspekte neben dem Slavischen und Slavistischen für den Lesestoff relevant bleiben. Aus dem Russischen fanden A. S. Puškin mit der Ballade Ribar in ri$b a$, ein Text zur altrussischen Geschichte nach N. Karamzin und eine Lebensskizze Deržavins Eingang in den Band. Miklosich selbst steuerte neben seiner Reisebeschreibung aus Konstantinopel auch polnische Sprichwörter (Slovensko Berilo 1853: 6) und ein Gedicht von J. Kochanowsky in slowenischer Übersetzung bei (22), außerdem schrieb er kurze Skizzen der tschechischen Nationallegenden in slowenischer Sprache (91-96) und verfasste ebenfalls in slowenischer Sprache die Lebensgeschichte des spätantiken Philsophen, Musiktheoretikers, Mathematikers und Staatsmannes Boëthius (Anicius Manilus Torquatus) Severinus (um 480-524) (127-130).

Der zweite Band der Reihe, das Lesebuch für die sechste Gymnasialklasse, hat in etwa dieselben Autoren. Der Entwicklung der Schüler Rechnung tragend, sind die Texte umfangreicher und anspruchsvoller. Historische und naturwissenschaftliche Beiträge sind in diesem Band stärker vertreten, die Lyrik tritt etwas zurück. Die mündlich überlieferte Sprachkunst ist durch das slowenische Heldenepos Pegam in Lambergar vertreten. ${ }^{8}$ Allerdings in einer Variante aus der Ausgabe von Emil Korytko

${ }^{8}$ Dies ist das erste in Druck erschienene slowenische epische Volkslied. Es war schon J. V. Valvazor und L. Schönleben bekannt gewesen. A. T. Linhart publizierte es als erster 1781 in seiner Sammlung Blumen aus Krain, allerdings in deutscher Übersetzung und in holprigen Hexametern. Valentin Vodnik erstellte eine Variante aus mehreren Aufzeichnungen. Nach dieser publizierte es mit einer literaturhistorischen Einleitung und parallelen deutschen Übersetzung 1806 und 1807 J. A. Zupančič: Der Turnier zwischen den beyden Rittern Lamberg und Pegam. Ein krainerisches Volkslied mit einer deutschen Übersetzung. Laibach 1807. 1850 nahm es Anastasius Grün in seine Volkslieder aus Krain auf. Wie beliebt und lebendig dieses Heldenepos unter dem slowenischen Volk gewesen war, davon zeugen die vielen Abbildungen des gegenständlichen Zweikampfes auch auf den Stirnbrettchen der Bienenstöcke. Alle im Laufe der Zeit bekannt gewordenen und gesammelten Varianten sind publiziert und kommentiert in: Slovenske ljudske pesmi. Prva knjiga. Pripovedne pesmi 1, ur. Zmaga Kumer, Milko Matičetov, Boris Merhar, Valens Vodušek, Ljubljana 1970, 5-15. 
(1813-1840). ${ }^{9}$ Dabei hat Miklosich nicht nur konsequent die Bohoričica in die Gajica umgesetzt, sondern auch kleinere sprachliche Korrekturen angebracht, so etwa Z. 3 Zefarost > Cesarost; Z. 8 fabó > seboj; Z. 9 tabó > teboj; Z. 9 De fe fkufit' sna $\int$ tabó $>$ Da se skusil bo s teboj; Z. 34 dalej $>$ dalje; Z. 38 roshnikranz $>$ roženkranc; Z. 46 pridrizhifh $>$ priderčiš $^{10}$. Erwähnenswert erscheint die Tatsache, dass Miklosich in diesen Band ein Gedicht des Kärntner Dichters Urban Jarnik (1784-1844) aufgenommen hat, und zwar Zvezde, das bereits zur slowenischen Vorromantik gehört und seine tiefempfundene spirituelle Gedankenlyrik repräsentiert (vgl. SturmSchnabl 2003: 43-62). Francè Prešeren ist mit seinen scharfen Epigrammen Seršeni vertreten. Miklosich selbst hat in diesem Band eine historische Skizze über die Herrscherin von Palmyra und der östlichen Reichsteile, Zenobia Cenobija, verfasst, ${ }^{11}$ in der er dem weiblichen Genie dieser Frau großen Respekt zollt.

Der dritte Band der Reihe, das Lesebuch für die siebente Gymnasialklasse erschien 1858. Das Titelblatt lautet: Slovensko Berilo za sedmi gimnazijalni razred. Izdal Dr. Fr. Miklošič. Velja 24 kr. C. M. Na Dunaju. V ces. kralj. zalogi bukev 1858. Den Löwenanteil der Texte und Übersetzungen schrieb J. Navratil, der bereits sehr intensiv mitgearbeitet hatte, da Miklosich zu dieser Zeit mit vielen Projekten befasst war. Im Jahre 1858 sind 13 Publikationen erschienen, darunter so arbeitsintensive und umfangreiche wie die Monumenta serbica spectantia historiam Serbiae, Bosnae, Ragusii oder der zweite Band seiner Zeitschrift Slavische Bibliothek oder

${ }^{9}$ Şlovénşke pesmi, krajnfkiga naróda. Zheterti svésik. V Ljubljani. Natisnil jih je, na prodaj jih ima Joshef Blasnik, na brégi, Nr. 190. 1841. Miklosich hatte sicherlich die Ausgabe von J. Zupančič gekannt, dieser war ja in Maribor im Gymnasium sein Lehrer gewesen. Doch war diese Variante eben erschienen und zwar in: Volkslieder aus Krain. Uebersetzt von Anastasius Grün. Leipzig. Weidmann'sche Buchhandlung 1850. Mit Anastasius Grün - Anton Alexander Graf Auersperg aber hatte Miklosich im Zusammenhang mit den Wahlen in das Frankfurter Parlament scharfe Polemiken geführt, die auch ihren schriftlichen Niederschlag fanden. Miklosich war als slowenischer Politiker gegen den Eintritt Österreichs ins Frankfurter Parlament, da er wie die meisten Slaven fürchtete, die Slaven der Monarchie würden damit völlig marginalisiert werden. Auch in seinem Manifest $Z e-$ dinjena Slovenija, das er vor seiner Wahl zum Reichstagsabgeordneten für die Steiermark im Namen des akademischen Vereins Slovenija in Wien verfasst hatte, formuliert er eine äußerst scharf argumentierte Absage an das Frankfurter Parlament, dessen brennender Verteidiger Anastasius Grün gewesen war (vgl. Granda 1991).

10 Diese wenigen ausgewählten Beispiele des normativen Eingreifens Miklosichs in den Konsolidierungsprozess der slowenischen Schriftsprache sollen nur der Illustration dienen. In Wirklichkeit findet sich die konsolidierende Normierung durchgehend in allen vier Lesebüchern für Gymnasien und betrifft die grammatischen Normen ebenso wie auch die Rechtschreibung. Martina Orožen, die sich in vielen Abhandlungen mit der Geschichte der slowenischen Schriftsprache befasst, hat bisher am klarsten den Anteil Miklosichs an der Konsolidisierung der slowenischen Schriftsprache in seiner Epoche herausgearbeitet. Vgl. Orožen 1996 u. 2003.

11 Zenobia Septimia, Fürstin von Palmyra 267-272. Führte nach der Ermordung ihres Mannes Odeanathus die Regierung für ihren Sohn Vaballathus und konnte ihr Herrschaftsgebiet bis nach Kleinasien, Arabien und Ägypten ausdehnen. Sie erklärte sich von Rom unabhängig und nahm den Titel Augusta an, wurde von Aurelianus bei Anthiochia besiegt und als Gefangene in Rom im Triumphzug mitgeführt. 
Beiträge zur slavischen Philologie und Geschichte (gemeinsam herausgegeben mit J. Fiedler). Miklosich bringt in diesem Band eine Sammlung serbischer Sprichwörter, die er ins Slowenische übersetzt hatte. Der keineswegs beschönigende Bericht von J. Rozman über den 4. Kreuzzug widerspiegelt die kritische Einstellung des Autors; dem entsprechend fällt auch der Bericht über den Fall Konstantinopels aus. Eine kleine biographische Skizze zu Jernej Kopitar verfasste J. Navratil, aus dessen Feder auch eine Kurzbiographie über Jurij Japelj auf der Grundlage einer Schrift von A. M. Slomšek stammt. Der Beitrag über die Čičen (Aromunen oder Walachen), die in Istrien in der so genannten Čičerija, deutsch Tschitschenboden, leben, ist eine Mischung von geographischen, ethnographischen und legendären Gegebenheiten; dieser Beitrag, Cičarija in pa Ciči [sic], stammt von Janez Bilec, nicht von Miklosich, der selbst später über diese Sprache und deren Interferenzen gearbeitet und publiziert hat. ${ }^{12}$ J. Terdina (Trdina) schreibt historische Beiträge über die Zustände in den slowenischen Ländern im 16. und 17. Jahrhundert. Zwei Erzählungen aus dem Serbischen sind ohne Angabe des Übersetzers und könnten von Miklosich stammen. Auch die naturwissenschaftlichen Beiträge behalten ihren Platz, stammen aber nicht mehr von J. Bleiweis.

Miklosichs vierter Band, das Lesebuch für die achte Gymnasialklasse, erschien 1865 und 1881 in einer zweiten, leicht veränderten Ausgabe, für die Miklosich J. Navratil die Redaktion anvertraute. Dieses Slovensko Berilo za osmi gimnazijalni razred. Izdal Dr. Fr. Miklošič. Vredil J. Navratil ist in zwei Teile gegliedert. Der erste Teil behandelt das Altkirchenslawische - stara slovenščina (Altslovenisch), ${ }^{13}$ das kyrillische Alphabet sowie einige kyrillische liturgische Textabschnitte, darunter die 58 Zeilen der Genesis mit einer interlinearen lateinischen Transkription. Danach werden die Freisinger Denkmäler behandelt, sodann das F II in extensu, abgedruckt mit einer interlinearen phonetischen Umschrift und einem Verzeichnis ungewöhnlicher Wörter mit lateinischer Übersetzung. Der zweite Teil, Neuslowenisch - nova slovenščina - ist nach Jahrhunderten eingeteilt und beginnt mit einem Bußgebet aus dem 15. Jahrhundert, das im Originalalphabet mit einer interlinearen Transkription in die Gajica abgedruckt ist. Für das 16. Jahrhundert werden die slowenischen Protestanten vorgestellt, hinzu kommen Textbeispiele aus dem Buch Moses. Für das 17. Jahrhundert werden der engagierte slowenische Gegenreformator Tomaž Hren (1560-1630), der Lexikograph Alasia da Sommaripa (um 1578-1626) ${ }^{14}$ und als Text

12 Die Aromunen, Walachen, Čičen oder Cinceri, ein romanisches, auf dem Balkan verstreut lebendes Volk, waren im 19. Jahrhundert in Istrien in der Čičerija/Tschitschenboden in geschlossenen Dörfern mit eigenen Pfarren angesiedelt und auch in einzelnen Orten des Küstenlandes zahlenmäßig relevant präsent. Miklosichs Abhandlungen zur Sprache und Kultur der Aromunen enthalten viel wertvolles Sprachmaterial (vgl. Sturm-Schnabl 1991: 164/4).

13 Zum Gebrauch der Termini stara slovenščina : nova slovenščina bei Miklosich vgl. Sturm-Schnabl 2004: 19-46.

14 Alasia da Sommaripa Alessandro, fra Gregorio aus Piemont wurde ins neugegründete Servitenkloster in Duino/Devin bei Monfalcone/Tržič berufen, um dieses und die ihm anver- 
eine slowenische Schwurformel aus der Steiermark vorgestellt. Das 18. Jahrhundert bevölkern aus regionaler Sicht die Krainer P. Marko Pohlin (1735-1801), Valentin Vodnik (1758-1819), Anton T. Linhart (1756-1795), Jurij Japelj (1744-1807) und Blaž Kumerdej (1728-1805), der Kärntner Oswald Gutsmann (1729-1790), Stefan Küzmič (1723-1779) aus Prekmurje sowie mit dem Kärntner Michael Andreaš (17621821) und dem Steirer Leopold Volkmer (1741-1816) auch zwei Bukovniki. Hinzu kommen wieder Textbeispiele. Das 19. Jahrhundert beginnt mit einer Darstellung von Leben und Werk Jernej Kopitars (1780-1844), interessant aber sind auch eine erste Biographie von Janez Žiga Popovič (1705-1774), ${ }^{15}$ eine umfassende Würdigung Anton Janežič' und andere Nekrologe von Männern der Wissenschaft und Literatur des 19. Jahrhunderts, darunter Urban Jarnik und Francè Prešeren. Von letzterem heißt es auf S. 59 des Lesebuchs: „Dr. France Prešeren je doslej najslavnejši pevec slovenski [....].“ Der junge Gregor Krek, später Slawist in Graz, wurde mit drei Gedichten aufgenommen. Alles in allem ist dieser 4. Band des Lesebuches für die 8. Gymnasialklasse so angelegt, dass den Schüler ein umfassendes slavistisches und vor allem slowenistisches Wissen angeboten wird. Unter den Textbeispielen überwiegen die slowenischen Originaltexte gegenüber Übersetzungen. Das slowenische Heldenepos Pegam in Lambergar wird hier noch einmal abgedruckt, diesmal in der Variante nach der Ausgabe von J. Župančič aus dem Jahre 1807. ${ }^{16}$ Auch in diesem Band sind Beiträge zu den einzelnen Regionen des slowenischen nationalen Territoriums abgedruckt, z. B. Železna Kapla in njena okolica na spodnjem Koroškem von O. Dular.

1850 entschloss sich Miklosich ein slowenisch-deutsches Wörterbuch herauszugeben. Das durch Jahre stetig gesammelte Sprachmaterial wollte er nun mit Hilfe seiner slowenischen Freunde und Informanten ergänzen. »Mislite na Francoze, kteri pravijo: Le dictionaire de la langue c'est le premier livre de chaque nation«, schreibt er an Jožef Muršec und ähnlich in einem Brief an Peter Kozler (vgl. Sturm-Schnabl 1991: 79-82). Dieses slowenisch-deutsche Wörterbuch wurde zu einer fast never ending story.

traute Pastorale zu organisieren. Selbst Sprachwissenschaftler, lateinisch und italienisch schreibender Schriftsteller, erlernte er die Sprache der dortigen slowenischen Bevölkerung und gab 1607 zur Unterstützung der pastoralen Tätigkeit ein italienisch-slowenisches Wörterbuch heraus. Diesem fügte er aus einer (verschollenen) Vorlage beschauliche slowenische Texte und fünf Kirchenlieder bei. Das Wörterbuch ist von Megisers Dictionarium Quattuor linguarum inspiriert, die Texte zeigen Merkmale der slowenischen Karstdialekte und Züge der damaligen slowenischen Schriftsprache der Protestanten (vgl. Da 5 Sommaripa 1979).

15 Janez Žiga Popovič, slowenischer Aufklärer, Lexikograph und Grammatiker. Professor für "deutsche Wohlredenheit" an der Universität Wien, berufen vom Wiener Erzbischof und Studienprotektor Joseph Fürst Trautson. Reutner hat den aktuellen Forschungs- und Wissensstand über Popovič vorbildlich aufgearbeitet und präsentiert (vgl. Reutner 2004: insbes. VII-CCXII).

16 Siehe Anm. 8 und 9. 
Das von Miklosich projektierte slowenisch-deutsche Wörterbuch ist als solches nicht erschienen. Er bemühte sich vor allem um 1850, das Material so weit als möglich zu vervollständigen. 1853 schreibt er an Bleiweis:

Wie viele Dinge fehlen uns noch! Uns fehlen vorzüglich jene, welche bei uns Bücher herauszugeben in der Lage sind. Eines der dringendsten Bedürfniße ist wohl ein Lexikon, da das Murkoische dem gegenwärtigen Stand der Sprachwissenschaft nicht entspricht. Ich habe viel darüber gearbeitet und einen über 300 Bogen umfaßenden Entwurf zu Stande gebracht ... Helfen Sie mir, wie man das Ding anfangen könnte, um zum Ziel zu gelangen. (Sturm-Schnabl 1991: 95)

Das gesammelte lexikalische Material stellte Miklosich für das Projekt eines großen slowenisch-deutschen Wörterbuchs zur Verfügung als Janez Zlatoust Pogačar (1811-1884) im Auftrag Jernej Vidmars (1802-1882), des Fürstbischofs von Ljubljana (1860-1872), die Herausgabe organisierte, deren Finanzierung der verstorbene Fürstbischof Anton Alois Wolf (1782-1859) aus seinem Nachlass sicher gestellt hatte. Die Finanzierung beinhaltete den Ankauf von Wörtersammlungen, die Redaktion und den Druck. Im Jahre 1865 wurde der Miklosich-Schüler Fran Levstik (1831-1887) zum Redakteur ernannt (vgl. Sturm-Schnabl 1991: 192/6, 194/11). Levstik wäre für Miklosich wohl der ideale Redakteur gewesen, da er wissenschaftlich dieselbe Meinung vertrat, was sich bereits in seiner Rezension zum deutsch-slowenischen Wörterbuch deutlich zeigte (vgl. Sturm-Schnabl 1991: 159). Uneigennützig stellte er dem Organisator sofort die vier Hefte seines Wörterbuchmanuskriptes zur Verfügung. Dem Bischof Vidmar ging es zu langsam, und Levstik wurde nach zwei Jahren entlassen. Erst als Janez Zlatoust Pogačar selbst Fürstbischof von Ljubljana geworden war, wurden die Arbeiten am Wörterbuch wieder aufgenommen. Nun wurde Maks Pleteršnik (1840-1923) zum Redakteur berufen. Wiederum überließ Miklosich sein Manuskript dem Fürstbischof Pogačar für das große Wörterbuchprojekt (vgl. Sturm-Schnabl 1991: 197-198).

Zur Zeit, als Pleteršnik die Redaktion inne hatte, wurden die vier Bände des Miklosich'schen Wortmaterials zwischen Wien und Ljubljana hin und her geschickt, da Miklosich selbst das Material zwischendurch für seine Arbeiten benötigte. ${ }^{17}$ Diese vier Hefte gingen dann verloren bzw. sind seither verschollen. Da Miklosich 1891 starb, als das Wörterbuch noch in Arbeit war, ist anzunehmen, daß dieses Material in Ljubljana geblieben ist. Die Bemühungen Miklosichs um ein slowenisch-deutsches Wörterbuch spiegeln sich jedenfalls seit 1850 in seiner Korrespondenz mit Peter Kozler, Jožef Muršec, Janez Bleiweis, A. Žakelj - Ledinski, Fran Levstik, J. Z. Pogačar und Maks Pleteršnik wider. Als das Wörterbuch im Jahre 1894 erschien, schildert Pleteršnik die Peripetien bei der Herausgabe des Wörterbuches, die verwende-

${ }^{17}$ Die kollegiale Zusammenarbeit und gegenseitige Hilfsbereitschaft spiegelt die Korrespondenz zwischen Miklosich und Pleteršnik wider (vgl. Sturm-Schnabl 1991: Br. 491, 514, $527,534,577,578)$. 
ten Wörtersammlungen und auch Miklosichs Bereitstellung seiner vier Hefte, die, wie Pleteršnik präzisiert, 287 Bögen umfassten. ${ }^{18}$

Schulbücher für Gymnasien, Grammatik, Wörterbuch, das waren konkrete Anliegen die Miklosich auf eine wissenschaftliche Basis stellte und so die Slowenistik als nationales Fach affirmierte. Was aber noch wesentlicher war, das waren seine Schüler, die einerseits als Mittelschullehrer tätig waren. Von den Slowenen seien genannt: Valenčak Martin (1835-1887), Šolar Janez (1827-1882), Šuman Josip (18381908), Klodič Anton (1836-1914), Nemanič Davorin (1850-1929) und Majciger Janez (1829-1909). Diese blieben meist zeitlebens seine Informanten in slavicis. Andere wiederum führten die Miklosich'sche Schule der Slowenistik sowohl wissenschaftlich als auch gesellschaftspolitisch weiter. Dazu gehören unter den Slowenen Valjavec Matija (1831-1897), Levstik Fran (1831-1887), Krek Gregor (1840-1905), Štrekelj Karel (1849-1912), Ferreri Klun Vinko (1823-1875), Murko Matija (18511952), Levec Franc (1846-1916), Pleteršnik Maks (1840-1923), Raič Anton (18451888) und Glaser Karel (1845-1913).

Der vorliegende Beitrag will und kann keinen Anspruch auf Vollständigkeit erheben, zu vielfältig ist die Bedeutung Miklosichs in zahlreichen Bereichen. Es handelt sich daher um eine Auswahl von Elementen, welche die Bedeutung Miklosichs für die Slowenistik an der Universität Wien sowie im europäischen Wissenschafts-, und, um einen modernen Begriff zu verwenden, Integrationsdiskurs aufzeigen.

Für den slowenischen Kulturraum wiederum hat Franz Miklosich durch die Herausgabe der Lesebücher für die Oberstufe der Gymnasien eine wesentliche Voraussetzung für die Heranbildung einer slowenischen Intelligenzia auf der Ebene der Mittelschulen geschaffen. Dies war neben dem konkreten bildungspolitischen Ziel auch eine seiner zahlreichen bedeutenden gesellschaftspolitischen Leistungen.

\section{Literatur}

Ceglar 1991: $\quad$ Charles A. Ceglar S. D. B., Bishop Frederic Baraga. The Works of, 1. Bd., Hamilton

Da Sommaripa 1979: Gregorij Alasia da Sommaripa, Slovar italijansko-slovenski, druga slovensko-italijanska in slovenska besedila, Videm 1607. Faksimile, Ljubljana - Devin-Nabrežina - Trst 1979 [Gregorio Alasia da Sommaripa, Vocabolario. Italiano-sloveno, altri testi italiano-sloveni e testi sloveni. Udine 1607. Liubiana - Duino-Auresina - Trieste 1979]

Granda 1999: $\quad$ Stane Granda, Prva odločitev Slovencev za Slovenijo. Dokumenti z uvodno študijo in osnovnimi pojasnili, Ljubljana

Mitrović 2001: $\quad$ Marija Mitrović, Geschichte der slowenischen Literatur. Von den Anfängen bis zur Gegenwart. Aus dem Serbokroatischen übersetzt, redaktionell bearbeitet und mit ausgewählten Lemmata und Anmerkun-

\footnotetext{
18 Slovensko-nemški slovar. Izdan na troške knezoškofa Ljubljanskega Antona Alojzija Wolfa. Uredil M. Pleteršnik. Prvi del A - O. V Ljubljani: Založilo in na svetlo dalo knezoškofijstvo. Tiskala katoliška tiskarna, 1894.
} 
gen ergänzt von Katja Sturm-Schnabl, Klagenfurt/Celovec - Ljublja-

Orožen 1996: na/Laibach - Wien/Dunaj

Martina Orožen, Uradovalno in pravno izrazje v Miklošičevem prevodu Občedržavljanskega zakonika, in: Oblikovanje enotnega slovenskega knjižnega jezika v 19. stoletju, Ljubljana, 229-246

Orožen 2003: $\quad$ Martina Orožen, Miklošičeva opredelitev slovnične zgradbe slovenskega jezika v primerjalnoslovanskih okvirih, in: dies.: Razvoj slovenske jezikoslovne misli (=Zora 26), Maribor, 160-172

Reutner 2004: Johann Siegmund Popowitsch, Vocabula Austriaca et Stiriaca. Nach der Abschrift von Anton Wasserthal herausgegeben und eingeleitet von Richard Reutner. Bd. 1-2, Frankfurt a. M.

Slovensko Berilo 1853: Slovensko Berilo za peti gimnazijalni razred. Izdal Dr. Fr. Miklošič. Velja 24 kr. C. M. Na Dunaju. V zalogi ces. kralj. bukev za šole pri Sv. Ani v Janezovih ulicah

Slovensko Berilo 1854: Slovensko Berilo za šesti gimnazijalni razred. Izdal Dr. Fr. Miklošič. Velja 24 kr. C. M. Na Dunaju. V zalogi ces. kralj. bukev za šole pri Sv. Ani v Janezovih ulicah

Slovensko Berilo 1858: Slovensko Berilo za sedmi gimnazijalni razred. Izdal Dr. Fr. Miklošič. Velja 24 kr. C. M. Na Dunaju. V ces. kralj. zalogi bukev

Slovensko Berilo 1865: Slovensko Berilo za osmi gimnazijalni razred. Izdal Dr. Fr. Miklošič. Vredil J. Navratil. Na Dunaju. Založil Karol Graeser $\left({ }^{2} 1881\right)$

Sturm-Schnabl 1991: Katja Sturm-Schnabl, Miklosich's Briefwechsel mit den Südslaven Miklošičeva korespondenca z južnimi Slovani, Maribor

Sturm-Schnabl 2003: Katja Sturm-Schnabl, Ideja romantične svobode in občutenja v poeziji Urbana Jarnika in Franceta Prešerna, Koroški etnološki zapisi 2 (Simpozij o Urbanu Jarniku. Zbornik predavanj), 43-62

Sturm-Schnabl 2004: Katja Sturm-Schnabl, Aktualnost Miklošičevega znanstvenega dela in misli, Jezikoslovni zapiski 10/2, 19-46

\author{
Katja Sturm-Schnabl \\ Institut für Slawistik der Universität Wien \\ Universitätscampus AAKH, Hof 3 \\ Spitalgasse 2, A-1090 Wien \\ katja.sturm-schnabl@univie.ac.at
}


